\title{
Hypertension research from Japan: advancing the field of renal denervation
}

\author{
Michael A. Weber ${ }^{1}$
}

Received: 24 November 2021 / Accepted: 24 November 2021 / Published online: 10 December 2021

(C) The Japanese Society of Hypertension 2021

The procedure of renal denervation offers an innovative approach for treating hypertension in patients whose blood pressures cannot adequately be controlled by lifestyle changes or medications. Interrupting the renal nerves not only decreases sympathetic stimulation of the kidneys but also reduces systemic sympathetic tone, thus providing two mechanisms by which blood pressure can be reduced. This new procedure has been undergoing clinical trials to test its efficacy and safety for over 10 years.

This issue of the journal publishes three important reports from Japan that are of worldwide importance. For a start, there is an article from Professor Kazuomi Kario and colleagues that addresses the important topic of patient preference [1]. In particular, would patients be willing to undergo a relatively minor invasive procedure if it offers the possibility of a needed reduction in blood pressure? The findings from this large survey are compelling and will be most helpful in positioning denervation in the hierarchy of medical treatments for hypertension. The second contribution from Dr. Yukako Ogoyama and colleagues is an up-todate meta-analysis of the efficacy and safety of renal denervation that have been demonstrated in the randomized controlled clinical trials completed globally to the present time [2]. Similarities and differences found among these trials are most illuminating. Again, this work provides a valuable resource for clinical scholars and public health experts to understand the place of denervation in patient management. And, finally, a further contribution from Professor Kazuomi Kario and his co-investigators reports the outcomes of REQUIRE, a large clinical trial performed in Japan that unexpectedly did not find the anticipated

Michael A. Weber

michaelwebermd@cs.com

1 Professor of Medicine, Division of Cardiovascular Medicine, State University of New York, Downstate Medical College, Brooklyn, NY, USA benefits of the renal nerve intervention [3]. But, as is sometimes the case, more can be learned from a study that does not meet its endpoints than from trials that are nominally successful. This important paper will have a major role in shaping the design of future research in this field.

\section{The patient preference survey}

The Japanese nationwide survey of patient attitudes towards renal denervation for treating hypertension provides an interesting perspective on key issues in achieving blood pressure control. In this survey of almost 2400 patients, all with previously diagnosed hypertension, currently receiving treatment and who could conduct home blood pressure mesurements, the study investigators were able to identify key predictors of willingness by patients to undergo the renal denervation procedure. This was not a comparison study, so we cannot draw conclusions about what patients might do if offered a choice between intensifying medication therapy or undergoing denervation to achieve blood pressure control. Still, about one-third of the hypertensive patients surveyed expressed a preference for the intervention. This finding was consistent with a survey on patients' attitudes to denervation reported previously from Germany [4].

There are some important new insights in this report. What were the predictors for the choice of the denervation procedure among Japanese patients? Not surprisingly, patients who had experienced side effects with their ongoing treatment, or whose blood pressures were not adequately controlled, were more likely to choose renal denervation. Of particular interest, in the relatively large proportion of patients who acknowledged that they were not fully adherent to their medication regimens, there was a greater interest in the procedure than by patients who were adherent to their treatment. This illustrates an important basic finding about hypertension management, for it shines light on the apparent paradox that patients who elect not to take their prescribed medications are nevertheless aware of the 
clinical risks of their inadequately controlled blood pressures. In fact, they are willing to undergo an invasive procedure to address these risks. It would be most interesting to study the reasons why patients-apparently knowledgeable about the dangers of persistent high blood pressure-decide to forgo simple medical therapy that in the current era is generally effective, well-tolerated, and inexpensive.

Of course, patients with relatively higher blood pressures were more likely than those with lower readings to select the preference for renal denervation. And, once again, confirming their understanding of the cardiovascular and stroke risks associated with uncontrolled hypertension and the need to find ways to bring their blood pressures down to safer levels.

It should be noted, however, that the average blood pressure of the patients in this Japanese cohort was 136/83 $\mathrm{mmHg}$ by office measurements and $135 / 83 \mathrm{mmHg}$ by home measurements. In fact, it was those patients with office blood pressures above $160 \mathrm{mmHg}$ or home blood pressures above $155 \mathrm{mmHg}$ who were most interested in renal denervation. So, it can be argued that many of the patients in this survey have close-to-normal blood pressures and so did not have a strong motivation to pursue alternatives to their current treatment. It is reassuring that the choice for denervation is strongest in patients whose needs are greatest.

These findings in Japanese patients cannot easily be compared with a survey of patient preference for renal denervation recently completed in the Unites States (so far available only in abstract form, presented at the recent Transcatheter Cardiovascular Therapeutics (TCT) meetings in Orlando, Florida). The American patients had far higher blood pressures_-averaging $155 \mathrm{mmHg}$ - despite receiving an average of approximately two antihypertensive drugs daily. Clearly these patients had a greater need for upgrading their hypertension treatment, whether by medication adjustments or renal denervation, than their Japanese counterparts. Indeed, based on the Japanese findings that about half of all patients with systolic blood pressures at $160 \mathrm{mmHg}$ or higher would be inclined to select renal denervation, we could infer that about half of the United States cohort would choose denervation. This inference actually is in harmony with what the patient preference study has shown in the United States, thus confirming a similarity in patient attitudes in the two countries.

In essence, what we have learned from this nationwide survey in Japan is that denervation would be seriously considered by approximately one-third of hypertensive patients currently taking medications. Overall, the findings from the Japanese survey suggest that renal denervation may be an acceptable alternative for a meaningful proportion of patients when treatment options are being weighed for optimizing hypertension treatment.

\section{Meta-analysis of controlled renal denervation trials}

It is important to get a sense of overall progress in the use of renal denervation. This procedure is a more focused and far better tolerated version of the surgical sympathectomies performed decades ago that reduced blood pressure quite effectively but left patients with major symptoms of autonomic dysfunction. Selectively destroying the renal nerves reduces blood pressure by ablating the efferent renal nerve fibers that stimulate the kidney; and, furthermore, by destroying the afferent fibers that run from the kidney to the central nervous system and thus reducing systemic sympathetic activity.

The meta-analysis by Professor Ogoyama now published in this journal provides a most valuable update of progress in this modality of hypertension treatment. The meta-analysis has focused on randomized blinded trials in which the effects on blood pressure of renal denervation have been compared with sham controls. As of now, nine such trials have been published, all using ambulatory blood pressure monitoring as the principal efficacy variable. Overall, in this new analysis 855 patients received denervation therapy and 670 were in the control groups. The mean reduction in $24 \mathrm{~h}$ systolic blood pressure, corrected by the sham effects, was a statistically significant $3.3 \mathrm{mmHg}$. Changes in daytime and nighttime effects were also significant, as was the mean office reduction of $5.3 \mathrm{mmHg}$.

Results in trials where hypertension patients were not taking medications ("off-meds" trials) were broadly similar in terms of efficacy to those in trials where patients were hypertensive despite taking medications ("on-meds" trials). As well, the methods used to create the nerve-ablating energy transmitted across the renal artery walls-either radiofrequency or ultra sound-produced roughly similar results.

Inevitably, there were differences among individual trials; in some studies the results were of relatively high magnitude, whereas in two trials $[3,5]$ the results were not significant. Even so, when these negative trials were included with the positive trials in the meta-analysis the overall efficacy result was positive and significant. It is important to acknowledge that differences can occur among trials, even when identical denervation procedures are used. The most recent trial to not provide the expected benefit of denervation, REQUIRE [3], is discussed below and provides valuable insights that will facilitate improvements in study design and conduct in the future.

Some interesting and unexpected findings regarding hypertension trials in general have emerged from the renal denervation experience. Publications from key studies have demonstrated the large variability in blood pressure responses among patients. This has occurred not only 
between the intervention and the sham groups, but within each of these groups. For instance, in the Spyral Off-Med study [6], fan plots showed how within both the intervention and the sham groups there was wide variability within individual patients-demonstrated by large increases and by large decreases in ambulatory blood pressure changes during the trial. In the sham group these large excursions tended to offset each other, whereas in the intervention group patients with large blood pressure reductions tended to outnumber those with increases, thus resulting in a modest but significant overall reduction [6]. Nevertheless, the intrinsic variability in ambulatory blood pressure measurements, presumably reflecting major differences in $24 \mathrm{~h}$ patient activity levels between the baseline and endpoint measurements, could easily obscure the therapeutic blood pressure effects of the denervation procedure.

One critical lesson from these observations is that clinical trials should include carefully conducted automated office measurements-similar to those performed in the SPRINT trial [7] - as a backup that might provide important additional information. Although ambulatory blood pressure monitoring is currently recommended as the standard primary endpoint measurement in hypertension clinical trials, its within-patient lack of reproducibility could potentially be a source of confounding. It is critical that this procedurewhich depends heavily on patients undergoing consistent activity levels on the days of measurement-be rigorously supervised both at baseline and at the study endpoint by clinical trial centers experienced in this work. It remains a matter of some concern that the intrinsic variability in $24 \mathrm{~h}$ blood pressure may actually be of greater amplitude than the effect size of the procedure being studied.

One question that often arises is: why are the blood pressure-lowering effects of renal denervation in open label trials so much greater than those in sham-controlled trials? In particular, the meta-analysis from Professor Ogoyama and colleagues reports an average $24 \mathrm{~h}$ systolic blood pressure reduction across multiple trials of around 3.3 $\mathrm{mmHg}$; whereas a recent open label study [8] and a major registry [9] report $24 \mathrm{~h}$ reductions that are closer to 10 $\mathrm{mmHg}$. Of course, the lesser effects in the controlled trials can be explained by the subtraction of the blood pressure effects in the sham groups, but this cannot fully explain the discrepancy between open label and sham studies. Findings from the trials in which there were excessive blood pressure reductions in the sham groups deserve close analysis, as exemplified by the recent REQUIRE trial [3] discussed below.

Finally, in discussing progress in the field of renal denervation there are two final points to be made. First, the relatively modest average effect size of the procedure of around $10 \mathrm{mmHg}$ by office readings in some of the key studies can legitimately be assumed to be predictive of meaningful reductions in event rates for major fatal and non-fatal cardiovascular outcomes [10]. Moreover, the blood pressure effects of denervation are sustained not just throughout the $24 \mathrm{~h}$ treatment period but have durability of at least 3 years. This is an attribute that can also be found with drug therapy, but only in patients who are strictly adherent to their treatment regimens-which, unfortunately, is often not the case [11]. The second point to be acknowledged is the marked heterogeneity of responses to the denervation procedure among patients $[6,12]$. It would be most helpful if we could identify patient characteristics that could identify those most likely to benefit. This vital issue awaits further research, as does further exploration of methods to measure the effectiveness of denervation in fully ablating the renal nerves, potentially providing real time guidance to operators during the procedure.

\section{Lessons from REQUIRE}

REQUIRE, published now in this journal, was a clinical trial performed in Japan in which the blood pressurelowering efficacy of endovascular ultrasound-mediated renal denervation was compared with a sham control [3]. This work was performed in parallel with a clinical trialParadise TRIO [13] — performed in the United States and Europe but supported by the same sponsor as REQUIRE and using the same ultrasound technology. Although both trials were performed in patients with high blood pressures despite taking medications, there was at least one major difference in study design. Whereas the patients in TRIO all received a 3-drug treatment regimen contained within a single pill, those in REQUIRE received a range of $1-8$ medications prescribed in a variety of regimens. Needless to say, the large number of drugs taken by many of these patients can, of itself, be considered as prima facie evidence of poor adherence to treatment, thus raising concern about inconsistent medication-taking throughout the trial [11]. And while TRIO had a successful conclusion, with highly significant blood pressure reductions when the intervention and sham arms were compared, the results in REQUIRE were far more modest, with the intervention and sham groups experiencing virtually identical reductions in their 24-hour systolic blood pressures and office readings.

This unexpected result in important ways resembled the experience in a previous trial-Symplicity-HTN 3 [14]—in which multiple in-trial changes in medication use in patients taking complex treatment regimens played a major part in confounding the results of the study and diluting the effects of renal denervation.

Perhaps the most interesting and illustrative blood pressure effects in REQUIRE, although not a pre-specified major endpoint, were provided by the home blood pressure 
values measured by the patients themselves [3]. By 1 month following the baseline procedures there was a significant $5.4 \mathrm{mmHg}$ difference in systolic blood pressure between the denervation and sham treatment arms. But, gradual ongoing reductions in blood pressure in the sham group, presumably reflecting intensification of medical therapy in some patients, and gradual ongoing blood pressure increases in the denervation group, presumably due to some patients reducing or discontinuing their medication therapy, diminished the difference between the groups to $3.8 \mathrm{mmHg}$ at 2 months and to only $1.8 \mathrm{mmHg}$ at 3 months [3]. These trends very likely were prompted by patients interpreting their own blood pressure values and making what they considered to be appropriate medication changes. Moreover, this unwanted situation might have been exacerbated by the interaction of study investigators who, unlike those in other denervation trials, were not blinded regarding the disposition of their patients to the intervention or sham groups and so may have "advised" patients in their medication adjustments. Further, the timing of medication use in relation to when blood pressures were measured was not standardized, introducing yet a further inconsistency in the trial's conduct.

But, despite this unexpected result, the REQUIRE trial has provided valuable information that will guide the trial that must now follow it. Quite clearly, "naturalistic" study designs, although conceptually attractive in some practice settings, cannot be the basis of trials where precise effects on blood pressure are critical endpoints.

As it is, the inconsistency of blood pressure measurements within patients and the variability created by uncertain medication adherence, highlight a compelling need to design trials in which these sources of confounding can be minimized. Previous studies for renal denervation set strict requirements for blood pressure measurements and great emphasis needs to be placed on achieving consistency of both ambulatory monitoring (admonishing patients to follow similar patterns of daily activity during the monitoring procedures throughout the trial) and carefully conducted automated office measurements. In addition, simplifying medication use into single pill combinations appears to be a critical step for enhancing adherence to treatment. And since the TRIO trial with its 3-drug regimen has now established the value of renal denervation in patients with resistant hypertension, the successor study to REQUIRE could validly be based on a slightly less aggressive 2-drug regimen which, although having strong antihypertensive properties, would nevertheless expand the range of patients eligible for entry to the trial and facilitate recruitment. And, in keeping with what has now become standard practice in these trials, confirmation of patients' medication-taking by laboratory testing as well as by witnessed pill-taking at critical study endpoints should be integrated into this research.
The meta-analysis and the patient preference studies discussed in this editorial both point to the need, value and acceptance of renal denervation in patients whose blood pressure are not adequately controlled by currently available treatment. And beyond that, experience from real-world settings $[8,9]$ fuels the hope that the benefits of this intervention may be even greater than those demonstrated in the sham-controlled trials reported so far. Inevitably, a critical further step will be the launch and completion of the forthcoming Japanese clinical trial of renal denervation now in advanced planning by an experienced group of researchers led by Professor Kario. Scientists and clinicians with an interest in the field of hypertension and the role of renal denervation will eagerly await the results of that important endeavor.

\section{Compliance with ethical standards}

Conflict of interest The author declares no competing interests.

Publisher's note Springer Nature remains neutral with regard to jurisdictional claims in published maps and institutional affiliations.

\section{References}

1. Kario K, Kagitani H, Hayashi S, Hanamura S, Ozawa K, Kanegae H. A Japan nationwide web-based survey of patient preference for renal denervation for hypertension treatment. Hypertens Res. 2021. https://doi.org/10.1038/s41440-021-00760-9.

2. Ogoyama Y, Tada K, Abe M, Nanto S, Shibata H, Mukoyama M, et al. Effects of renal denervation on blood pressures in patients with hypertension: a systematic review and meta-analysis of randomized sham-controlled trials. Hypertens Res. 2021. https:// doi.org/10.1038/s41440-021-00761-8.

3. Kario K, Yokoi Y, Okamura K, Fujihara M, Ogoyama Y, Yamamoto E, et al. Catheter-based ultrasound renal denervation in patients with resistant hypertension: the randomized, controlled REQUIRE trial. Hypertens Res. 2021;397:1-11.

4. Schmieder RE, Högerl K, Jung S, Bramlage P, Veelken R, Ott C. Patient preference for therapies in hypertension: a cross-sectional survey of German patients. Clin Res Cardiol. 2019;108:1331-42.

5. Bhatt DL, Kandzari DE, O'Neill WW, D'Agostino R, Flack JM, Katzen BT, et al. A controlled trial of renal denervation for resistant hypertension. N Engl J Med. 2014;370:1393-401.

6. Townsend RR, Mahfoud F, Kandzari DE, Kario K, Pocock S, Weber MA, et al. Catheter-based renal denervation in patients with uncontrolled hypertension in the absence of antihypertensive medications (SPYRAL HTN-OFF MED): a randomised, shamcontrolled, proof-of-concept trial. Lancet 2017;390:2160-70.

7. SPRINT Research Group, Lewis CE, Fine LJ, Beddhu S, Cheung AK, Cushman WC, Cutler JA, et al. Final report of a trial of intensive versus standard blood-pressure control. N Engl J Med. 2021;384(May):1921-30. https://doi.org/10.1056/NEJMoa1901281.

8. Mahfoud F, Sievert H, Bertog S, Lauder L, Ewen S, Lengelé JP, et al. Long-term results up to 12 months after catheter-based alcohol-mediated renal denervation for treatment of resistant hypertension. Circ Cardiovasc Inter. 2021;14:e010075. https://doi. org/10.1161/CIRCINTERVENTIONS.120.010075.

9. Mahfoud F, Bohm M, Schmieder R, Narkiewicz K, Ewen S, Ruilope L, et al. Effects of renal denervation on kidney function 
and long-term outcomes: 3-year follow-up from the Global SYMPLICITY Registry. Eur Heart J. 2019;40:3474-82.

10. Ettehad D, Emdin CA, Kiran A, Anderson SG, Callender T, Emberson $\mathrm{J}$, et al. Blood pressure lowering for prevention of cardiovascular disease and death: a systematic review and metaanalysis. Lancet 2016;387(Mar):957-67. https://doi.org/10.1016/ S0140-6736(15)01225-8.

11. Azizi M, Pereira H, Hamdidouche I, Gosse P, Monge M, Bobrie G, et al. Adherence to antihypertensive treatment and the blood pressure-lowering effects of renal denervation in the renal denervation for hypertension (DENERHTN) trial. Circulation 2016;134:847-57.
12. Azizi M, Schmieder RE, Mahfoud F, Weber MA, Daemen J, Davies $\mathrm{J}$, et al. Endovascular ultrasound renal denervation to treat hypertension (RADIANCE-HTN SOLO): a multicentre, international, singleblind, randomised, sham-controlled trial. Lancet 2018;391:2335-45.

13. Azizi M, Sanghvi K, Saxena M, Gosse P, Reilly JP, Levy T, et al. Ultrasound renal denervation for hypertension resistant to a triple medication pill (RADIANCE-HTN TRIO): a randomised, multicentre, single-blind, sham-controlled trial. Lancet 2021. https:// doi.org/10.1016/S0140-6736(21)00788-1.

14. Bhatt DL, Kandzari DE, O'Neill WW, D'Agostino R, Flack JM, Katzen BT, et al. A controlled trial of renal denervation for resistant hypertension. N Engl J Med. 2014;370:1393-401. 